
\title{
25 Research Square \\ Total Cardiovascular Risk in the Elderly Population of Spain: The Epicardian Risk Score
}

Rafael Gabriel ( $\nabla$ rgabriel@ceiis.org )

Instituto de Salud Carlos III

Javier Muñiz

Universidade da Coruna

Saturio Vega

Gerencia Regional de Salud

Irene Moral

EAP Sardenya

Isidro Lopez

Servicio Galego de Saude

Teresa R. Pérez Castro

Servicio Galego de Saude

Francisco Rodriguez-Salvanés

Hospital Universitario de la Princesa

Carmen Suárez

Hospital Universitario de la Princesa

Blanca Novella

Hospital Universitario de la Princesa

Carlos Brotons

EAP Sardenya

Research article

Keywords: cardiovascular risk estimation, elderly, CVD

Posted Date: June 25th, 2020

DOl: https://doi.org/10.21203/rs.3.rs-33921/v1

License: (a) (i) This work is licensed under a Creative Commons Attribution 4.0 International License.

Read Full License 


\section{Abstract}

Background: Cardiovascular risk estimation in people over 70 years is problematic. Most scores have been derived from cohorts of middle-aged people, with older persons under-represented. The predictive power of classical cardiovascular risk factors reduce with age. The aim is to develop a specific score for the elderly population

Methods: Population-based cohort established in 1995. Setting: Three geographical areas of Spain (Madrid, Ávila and Lugo). Participants: 3,729 people older than 64 years, free of any cardiovascular diseases (CVD), at baseline. Measurements: Suspected fatal and nonfatal CVD (both coronary heart disease and stroke) were yearly investigated and confirmed using the WHO-MONICA criteria. All participants were followed until the occurrence of the first CVD event, until death or until December 31th 2015 if alive.

Results: Age was the strongest predictor of 10-year CVD both in men and women. In men, variables associated to CVD were high blood pressure treatment (HR: 1,35; 95\% Cl: $1,067,1,710)$, diabetes (HR: 1,359; 95\% Cl: 0,997, 1,852) and smoking (HR:1,207; 95\%Cl: 0,945, 1,541), and in women smoking (HR: 1,881 95\%Cl: 1,356, 2,609) and diabetes (HR:1,285; 95\%Cl:0,967, 1,707). Total-cholesterol did not increased the risk of CVD either in males or females. However, total-cholesterol level $(>200 \mathrm{mg} / \mathrm{dl})$ were negatively associated both in men and women.

Conclusions: In old Spanish men, 10-year total CVD is significantly increased by age, diabetes and antihypertensive treatment, and in old women by diabetes and smoking. Total-cholesterol levels did not increased the risk of CVD either in males or females.

\section{Background}

Currently, several Cardiovascular Disease (CVD) risk charts are available, but the majority have been developed from middle-aged populations with few individuals older than 70 years represented $[1,2]$. In addition the existing scores have several limitations for their use in the elderly, because usually do not consider the effect of cardiovascular risk factors (CVRF) treatment [3] or frailty, both important determinants of CVD in older persons, and therefore can wrong estimate the actual CVD risk $[4,5]$. For example, the European Score concentrates mainly on the middle-aged group, and recommends it use in the range of 40 to 65 years [1].

Moreover, observational studies in the elderly have shown that risk estimation in people older than 70 years is problematic because the predictive power of CVRF is reduced with age. The Leiden 85-plus study showed that Framingham score is not useful for people older than 70 , because of its low discrimination power [6].

Also, several studies have reported that the association of hypertension and mortality is attenuated in older adults, and therefore age might be a poor determinant of hypertension and CVD in the elderly [7- 
10]. Measures of frailty and functioning may however identify better old persons at risk for the adverse consequences of hypertension. They found that SBP is associated with an increased risk of mortality in elders with reduced walking speed [11]. In addition, some classical CVRF as total-cholesterol (TC) become nebulous, or even act in the reverse direction, at older ages [12]. Thus, a more adequate assessment of CVD risk in the old and very old is desirable in order to target and optimize prevention and treatment.

The aim of this study is to determine the major predictors for total CVD risk in the Spanish elderly, and to develop specific CVD risk charts for this age-group.

\section{Methods}

The EPICARDIAN is a multicenter, population-based, cohort study in people 65 years and older from three geographical areas of Spain: Madrid city, Arévalo county (Ávila province) and Begonte county (Lugo province). Study design and methods have been previously published elsewhere [13, 14]. In summary a total of 3,729 individuals older than 64 years participated in the baseline examination. Two hundred fifty five cases (6.8\%) shown overt clinical CVD at baseline. Only individuals free of CVD at baseline were included in the analyses. Therefore the inception cohort was composed by 3,474 individuals $(1,462$ men and 2,012 women). All participants were followed until the occurrence of first cardiovascular event, death or 10-years if alive.

Variables measured at baseline and included in the risk function were age, sex, SBP, smoking, totalcholesterol, fasting glucose, medical diagnosis of hypertension, hypercholesterolemia, diabetes mellitus, and specific drug treatment for these conditions. Serum total-cholesterol was determined by enzymatic method [15]. LDL-cholesterol was not estimated in the cohort because only a small proportion of participants had HDL-cholesterol values available. Plasma glucose was determined by the glucoseoxidase method [16]. For the Cox regression analysis, variables were grouped using the Framingham risk factor categories [17]: age was grouped in five-year categories starting from 65-69 years, SBP: in three and TC: in four. Smoking was categorized as smokers (those who smoked at baseline or quitted smoking less than 6 months), and non-smokers/ex-smokers the rest (table 1). Hypertension was defined if SBP $\geq 140 \mathrm{mmHg}$ or $\mathrm{DBP} \geq 90 \mathrm{mmHg}$, or taking antihypertensive treatment [18]. Hypercholesterolemia if $\mathrm{TC} \geq 5.1 \mathrm{mmol} / \mathrm{L}$ or taking lipid-lowering treatment [15] and Diabetes if fasting plasma glucose $\geq 7.0 \mathrm{mmol} / \mathrm{L}$, or taking antidiabetic treatment [19].

Information on CVD events in the inception cohort was annually seek from primary care and hospital records, general practitioners, postal mail, and phone calls to the subject or relatives Date and cause of death of deceased individuals in the cohort were confirmed through the Spanish National Death Index and the mortality registries of each health area.

The International Classification of Diseases (ICD-9 and ICD-10), was used for registration of coronary heart disease (ICD-9: 410-414, ICD-10: I20-I25) and other cardiovascular conditions (ICD-9: 797-799; ICD10: 146, R96, R98, I50). Coronary events were confirmed using the WHO-MONICA algorithm [20]. CVD 
outcome was defined as a composite of first coronary event (fatal or non-fatal definite Myocardial Infarction (MI) and possible-fatal MI).

For description of the selected data, arithmetic mean with standard deviation (SD) was used when the variables were continuous and relative frequency distribution when categorical. For comparison of proportions, the Pearson's chi-squared $\left(\chi^{2}\right)$ test and the Sommer's D were used in the case of ordinal variables. For group comparison, the Student-Fisher $t$ test was used in the case of independent binary measures and analysis of variance for variables of more than 2 categories.

For the estimation of general risk function and multivariate analysis 10-year total (fatal and non-fatal) CVD and competitive 10-year total mortality from non-CVD deaths.

Described independent variables were included in the multivariate models using the ENTER method. We estimated separate models for males and females. In a first step we managed SBP and TC as continuous variables in order to assess interactions between antihypertensive treatment and SBP values, and between lipid lowering drug and TC levels. In a second step SBP and TC were categorized. In the third step the interactions were withdrawn. Finally in order to simplify the model, the variable lipid lowering treatment was deleted because was not statistically significant.

Bivariate and multivariate associations between each independent variable in the model and the outcome were assessed. ß-coefficient associated with each potential CVRF and the first ever recorded CVD event were estimated by Cox regression analysis, separately for men and women. Ten-year Kaplan-Meier functions for first fatal/non-fatal CVD events were derived to calculate the mean cumulative incidence. The estimate of survival function was performed using the Cox method stratified by sex. Omnibus test was used to validate the model $(p<0.05)$. Sex-specific risk score sheets for prediction of fatal and nonfatal CVD using categorical variables were developed from the $\beta$-coefficients of Cox proportional hazards models. The observed 10-year total CVD risk was categorized in low-risk (<20\%), mild-risk $(20-29 \%)$, moderated-risk (30-39\%), moderated-high risk (40-49\%), high-risk (50-59\%) and very-high risk ( $\geq 60 \%)$.

\section{Results}

The 3,474 participants contributed with 27,749 person-years of follow-up. At baseline $42.5 \%$ of the sample had hypertension; $28.4 \%$ hypercholesterolemia, $11.5 \%$ diabetes mellitus, $11.9 \%$ smoked and $52 \%$ were on antihypertensive treatment. Follow-up information was obtained in 3,474 persons $(99.8 \% ; 1,462$ men and 2,012 women). There were a total of 1,340 deaths $(38,6 \%)$ in the entire cohort during the followup (702 deaths in men and 638 in women), with 457 fatal-CVD death (222 in men, and 235 in women). In total were observed 768 CVD events ( 457 fatal-CVD events and 311 non-fatal CVD events. Table 1 shows cardiovascular CVRFs and CVD events distribution by gender.

Table 2 shows multivariate Cox hazard models of 10-year average first fatal or non-fatal CVD for men (panel A), and women (panel B). Age was the strongest predictor of CVD both in men and women. In men, the stronger variables associated to CVD were: high blood pressure treatment (HR: 1,35; 95\% Cl: 1,067, 
1,710), diabetes (HR: 1,359; 95\% Cl: 0,997, 1,852) and smoking (HR:1,207; 95\%Cl: 0,945, 1,541). In women the stronger variables associated to CVD were smoking (HR: $1,88195 \% \mathrm{Cl}: 1,356,2,609)$ and diabetes (HR:1,285; 95\%Cl:0,967, 1,707). The model showed a discriminative power (C-statistic) of 0.656 in men and 0.702 in women.

In a second step analysis we used age as a time-scale variable, rather than as a typical independent variable. The Cox model significantly improved for diabetes prediction. The HR of diabetes increased from 1, 37 to 1, 48 in men; and from 1, 28 to 1, 34 in women (data not shown). HR of other variables considered in the Cox model (serum total-cholesterol, smoking, SBP or antihypertensive treatment) did not increase when age was handled as time-scale.

Table 3 shows the individual 10-year absolute cardiovascular risk specifically for smoker's men (panel A) and non-smoker's men (panel B); Table 4 shows the corresponding values for smoker's women (panel A) and non-smoker's women (panel B). This visual format facilitates the reading of 10-year individual absolute total cardiovascular risk (fatal and non-fatal CVD) for each gender-smoking status, taking also into account the antihypertensive treatment, diabetes status, age, TC and SBP level of each individual.

\section{Discussion}

The EPICARDIAN-score is based on a large cohort of 3,474 old Spanish citizens. Previous scores as Framingham and European SCORE are based on population recruited long time ago, before the 1980s, when the percent of risk factors was lower at population level. In contrast the EPICARDIAN cohort was assembled more recently and provides risk estimation for people treated with and without antihypertensive and other cardiovascular drugs. The EPICARDIAN-score overcomes also the problem of applying ß-coefficients derived from studies in middle-aged populations to older persons, which may be inadequate for risk estimation in the elderly.

Age is the strongest predictor of cardiovascular risk in older men and women. However, when using age as a time-scale variable, rather than as a standard variable, the performance of Cox model improved, particularly for women. For example, diabetes emerged as an independent predictor in women (in the original model diabetes was borderline).

A different CVRF pattern was observed in males and females. In men, diabetes was the strongest predictive factor, and high blood pressure treatment had a greater impact than in women. Smoking shown also a significant effect on CVD, but it was higher in women than in men. Similar to previous studies [21, 22], the contribution of TC to CVD act in the reverse direction after the age of 70 . The absence of a positive predictive value for TC raises the concept that risk estimation in the elderly may warrant a different approach than in middle-aged individuals, and confirms the predictive power of certain classic risk factors as hypercholesterolemia diminish with age.

Current charts used to predict CVD in old people mainly rely on calibrated risk function from the original Framingham [23] and the European scores [24]. Important limitations of these scores are the small 
number of people older than 70 years, the occupational origin of some cohorts, and the consideration of CVD mortality as the only main outcome in the predictive model. In contrast, the EPICARDIAN score includes an important proportion of individuals older than 75 years, and provides total CVD risk, a more comprehensive estimate of the individual total risk in a Mediterranean country as Spain, with low rates of CHD. Moreover, previous European charts for the elderly do not include diabetes or, as the Framingham algorithm, prediction is based on a small number of diabetic people (4\%), not based on the current International definition of diabetes. Finally, the EPICARDIAN-charts uniquely accounted for the use of antihypertensive drugs, the most common treatment in older people, which allows solving a major gap in previous risk assessment tools [25].

Recently, a new European SCORE system for old persons, SCORE-OP, has been published [26]. We applied the SCORE-OP to our population, and found the 10-year average CVD risk was significantly lower than the obtained with the EPICARDIAN charts, both in men and women [27]. This is most probably due to the fact that the mean age of the EPICARDIAN population is older, and the prevalence of diabetes and smoking higher than in the cohorts included in the SCORE-OP, mainly coming from Northern and Central Europe. Also, the two equations do not include exactly the same parameters. For instance the EPICARDIAN equation includes hypertension treatment as a variable in the model, while the SCORE-OP includes C-HDL. Finally we also have shown that the SCORE-OP identifies fewer patients with 65 year and older, at high or very high risk, than the original derived SCORE function for low-risk countries, as Spain [27].

The EPICARDIAN charts consider only the major CVRF. Other factors as family history of CVD, fibrinogen level, physical activity, waist circumference or C-reactive protein, can theoretically modulate the CVD risk in the elderly. In contrast, as mentioned above the predictive value of classic risk factors, as hypercholesterolemia or systolic hypertension, weakens with age [8, 21], and the absolute risk estimations in some cells, particularly in those groups of older age with few individuals with very high levels of TC and SBP could be less accurate because of this phenomenon.

Our study has also strengths, as a sample recruited from urban and rural settings, different geographical locations, wide range of old ages, and large proportion of diabetic individuals. The EPICARDIAN cohort provides the first prospective association between total CVD and several CVRFs for the elderly in Spain, a country with low incidence of CVD. The EPICARDIAN system allows the direct estimation of total CVD risk based on the background contemporaneous risk of the elderly population, rather than based in foreign scores calibrated for low risk European population. Predictions based on these calibrations may not work if a long time and a marked change in CVD mortality occurred between the risk score was derived and applied to different populations.

\section{Conclusions}

Our results show the extent to which the effect of some specific CVRFs as cholesterol levels, maybe assessed in the specific population to which they are going to be applied. It is expected that validation of this risk score will be done in the future. The availability of information on CVRF treatment, particularly 
antihypertensive, is another strength of this study, and a useful information for clinicians to adjust the estimated CVD risk in those patients already under antihypertensive treatment.

The EPICARDIAN score offers a novel estimation of total CVD risk incorporating the hypertensive treatment to the risk calculation. CVD risk is significantly increased by diabetes and antihypertensive treatment in older men and by smoking in older women. The score is a practical tool for clinicians to estimate the total individual CVD risk in Spanish older than 70 years.

\section{Abbreviations}

CVD: Cardiovascular Disease

CVRF: Cardiovascular Risk Factor

HR: Hazard ratio

Cl: Confidence interval

SBP Systolic Blood Pressure

DBP Diastolic Blood Pressure

TC Total Cholesterol

CHD: Coronary Heart Disease 11or na Gabrila from ....id and m....To Diana Gabrila from ....her useful commentszo por statistical assistance

\section{Declarations}

Ethics approval and consent to participate: The study is in compliance with the Helsinki Declaration (http://www.wma.net/e/policy/b3.htm). We obtained written informed consent of all participants at baseline examination. The study protocol was approved by the Ethic Committee of La Princesa University Hospital, Madrid.

Consent for publication: All authors given personal written consent for publication of this manuscript.

Availability of data and material: All data and materials are open available upon request.

Competing interests: No competing interests for none of the authors.

Funding: FIS 93/012; FIS 96/1993; FIS 02/0896 from Instituto de Salud Carlos III and DGICyT PB/255Co4. Spain. The funder(s) had no role in study but funding.

Authors' contributions: RG principal investigator, study design , analysis \& manuscript drafting;' JM: ' study design , analysis \& manuscript drafting SV: field works, data collection, manuscript drafting; IM 
statistical analysis; IL field works, data management; TRPC: field works, data management FRS statistical analysis, manuscript drafting; CS study design , analysis \& manuscript drafting; BN analysis \& manuscript drafting CB study design, analysis \& manuscript drafting.

Acknowledgements: To Charo Madero from Biostatistics Unit of Hospital Universitario La Paz, Madrid for statistical advice.

\section{References}

1. Conroy RM, Pyörälä K, Fitzgerald AP, Sans S, Menotti A, De Backer G, et al. SCORE project group. Estimation of ten-year risk of fatal cardiovascular disease in Europe: the SCORE project. Eur Heart $J$ 2003; 24:987-1003

2. Gabriel R, Brotons C, Tormo MJ, Segura A, Rigo F, Elosua R. ERICE study group. The ERICE-score: the new native cardiovascular score for the low-risk and aged Mediterranean population of Spain. Rev Esp Cardiol (Engl Ed). 2015;68:205-15

3. Liew SM, Doust J, Glasziou P. Cardiovascular risk scores do not account for the effect of treatment: a review. Heart 2011;97:689-97

4. Cooney MT, Dudina AL, Graham IM. Value and Limitations of Existing Scores for the Assessment of Cardiovascular Risk. A Review for Clinicians. J Am Coll Cardiol 2009; 54:1209-27

5. Hoes AW, Grobbee DE, Valkenburg HA, Lubsen J, Hofman A. Cardiovascular risk and all cause mortality: a 12-year follow-up study in The Netherlands. Eur J Epidemiol. 1993; 9:285-92

6. deRuijter W, Westendorp RG, Assendelft WJ, den Elzen WP, de Craen AJ, le Cessie S, et al. Use of Framingham risk score and new biomarkers to predict cardiovascular mortality in older people: population based observational cohort study. BMJ. 2009; 338:a3083.

7. Satish S, Freeman DH Jr, Ray L, Goodwin JS. The relationship between blood pressure and mortality in the oldest old. J Am Geriatr Soc. 2001; 49:367-74

8. Bemmel T, Gussekloo J, Westendorp RG, Blauw GJ. In a population-based prospective study, no association between high blood pressure and mortality after age 85 years. J Hypertens 2006;24:28792

9. Oates DJ, Berlowitz DR, Glickman ME, Silliman RA, Borzecki AM. Blood pressure and survival in the oldest old. J Am Geriatr Soc 2007; 55:383-8

10. Rastas S, Pirttila T, Viramo P, Verkkoniemi A, Halonen P, Juva K, et al. Association between blood pressure and survival over 9 years in a general population aged 85 and older. J Am Geriatr Soc 2006; 54:912-8

11. Odden MC, Peralta CA, Haan MN, Covinsky KE. Rethinking the Association of High Blood Pressure with Mortality in Elderly Adults: The Impact of Frailty. Arch Intern Med. 2012; 172:1162-68

12. Weverling-Rijnsburger AW, Blauw GJ, Lagaay AM, Knook DL, Meinders AE, Wetendorp RG. Total cholesterol and risk of mortality in the oldest old. Lancet 1997; 350:1119-23 
13. Novella B, Alonso M, Rodriguez-Salvanés F, Susi R, Reviriego B, Escalante L, et al. Ten-year incidence of fatal and non-fatal myocardial infarction in the elderly population of Madrid. Rev Esp Cardiol 2008; 61:1140-9

14. Gabriel R, Alonso M, Reviriego B, Muñiz J, Vega S, López I, et al. Ten-year fatal and non-fatal myocardial infarction incidence in elderly populations in Spain: the EPICARDIAN cohort study. BMC Public Health. 2009; 9:360-8

15. Ray KK, Kastelein JJ, Boekholdt SM, Nicholls SJ, Khaw KT, Ballantyne CM, et al. The ACC/AHA 2013 guideline on the treatment of blood cholesterol to reduce atherosclerotic cardiovascular disease risk in adults: the good the bad and the uncertain: a comparison with ESC/EAS guidelines for the management of dyslipidaemias 2011. Eur Heart J. 2014;35:960-8

16. Carstensen B, Lindström J, Sundvall J, Borch-Johnsen K, Tuomilehto J; DPS Study Group. Measurement of blood glucose: comparison between different types of specimens. Ann Clin Biochem 2008; 45:140-8

17. Wilson PW, D’Agostino RB, Levy D, Belanger AM, Silbershatz H, Kannel WB. Prediction of coronary heart disease using risk factor categories. Circulation. 1998; 97:1837-47

18. Chobanian AV, Bakris GL, Black HR, Cushman WC, Green LS, Izzo JL Jr, et al. The Seventh Report of the Joint National Committee on Prevention, Detection, Evaluation, and Treatment of High Blood Pressure. The JNC 7 Report. JAMA 2003; 289:2560-72

19. World Health Organization. Definition and diagnosis of diabetes mellitus and intermediate hyperglycemia: report of a WHO/IDF consultation. WHO, 2006

20. Tunstall-Pedoe H; Kuulasmaa K, Amouyel P, Arveiler D, Rajakangas AM, Pajak A.. Myocardial infarction and coronary death in the World Health Organization MONICA Project. Registration procedures, event rates and case-fatality rates in 38 populations from 21 countries in four continents. Circulation 1994; 90:583-612

21. Tuikkala P, Hartikainen S, Korhonen SM, Lavikainen P, Kettunen R, Sulkava R, et al. Serum total cholesterol levels and all-cause mortality in a home-dwelling elderly population: a six-year follow-up. Scand J Prim Health Care 2010; 28:121-127

22. Tilvis R, Valvanne JN, Strandberg TE, Miettinen TA. Prognostic significance of serum cholesterol, lathosterol, and sitosterol in old age; a 17-year population study. Annals of Medicine 2011;43:292301

23. Kannel WB. Coronary heart disease risk factors in the elderly. Am J Geriatr Cardiol 2002; 11:101-7

24. Sans S, Fitzgerald AP, Royo D, Conroy R, Graham I. Calibrating the SCORE Cardiovascular Risk Chart for Use in Spain. Rev Esp Cardiol 2007; 60:476-85

25. McGovern PG, Pankow JS, Sharar E, Doliszny KM, Folsom AR, Blackburn H, et al. Recent trends in acute coronary heart disease-mortality, morbidity, medical care, and risk factors. N Engl J Med 1996; 334:884-90

26. Cooney MT, Selmer R, Lindman A, Tverdal A, Menotti A, Thomsen T, et al; SCORE and CONOR investigators. Cardiovascular risk estimation in older persons: SCORE OP. Eur J Prev Cardiol. 2016; 
23:1093-103

27. Brotons C, Moral I, Fernández D, Cuixart L, Soteras A, Puig M. Assessment of the New SCORE OP Cardiovascular Risk Charts in Patients Older Than 65 Years. Rev Esp Cardiol (Engl Ed) 2016; 69:9813

\section{Supplementary Files}

This is a list of supplementary files associated with this preprint. Click to download.

- Tables.docx 